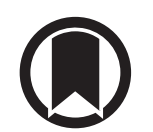

CrossMark

\title{
Home spirometry in bronchiolitis obliterans after allogeneic haematopoietic cell transplant
}

\author{
To the Editor:
}

Bronchiolitis obliterans syndrome (BOS) is a well-characterised late-onset noninfectious pulmonary complication of allogeneic haematopoietic stem cell transplantation (HSCT), occurring in $2 \%$ to $26 \%$ of recipients [1]. It is considered to be a pulmonary manifestation of graft versus host disease (GVHD) [2] and is associated with a mortality rate varying from $14 \%$ to $100 \%$ in historical series [3, 4]. Although immunosuppressive drugs are modestly effective, early diagnosis and treatment could improve its outcome [5]. After lung transplantation, home spirometry monitoring of pulmonary function allows early detection of BOS [6]; it is associated with a better response to steroids [7] and consequently is considered as a standard of care [8]. Our group was the first to report the use of home spirometry in 37 HSCT recipients [9]. More recently, CHENG et al. [10] showed a good correlation between home spirometry and classical laboratory spirometry in this population. We present here the incidence and long-term outcome of BOS occurring after HSCT in an extended cohort of 110 patients monitored with home spirometry.

This single-centre study was conducted in 110 patients who received an allogeneic HSCT for haematological malignancy from June 2001 to November 2008. Inclusion criteria were: 1) living in the Paris area, 2) having a landline telephone and 3) consenting to the study. BOS was defined as a persistent non-reversible obstructive ventilatory impairment, with a drop of forced expiratory volume in $1 \mathrm{~s}$ (FEV1) above $20 \%$ compared to baseline value before the graft, after exclusion of other causes, using the International Society of Heart and Lung Transplantation criteria [11]. No histological confirmation was required due to the high risk of the procedure in this population.

Before HSCT, complete pulmonary functional tests (PFT) were performed and patients were trained to perform flow-volume curves twice a week with home spirometry (individual portable spirometer, Spirotel, M-Elect, France, and its modem). home spirometry monitoring was scheduled to start three months after transplantation for 18 months. home spirometry results were centrally transmitted in real time using a landline telephone for analysis and monitoring. For each patient, correlation between data from home spirometry and from classical laboratory spirometry was checked before transplant. When home spirometry deterioration was detected, with a $\mathrm{FEV} 1$ drop superior to $20 \%$, patients were referred to pulmonologists for BOS diagnosis by PFT confirmation and performance of a high-resolution thoracic computed-tomography scan with expiratory phases and an infectious workup including a fiberoptic bronchoscopy with bronchoalveolar lavage to rule out a pulmonary infection. The trial was conducted in accordance with the declaration of Helsinki.

Main characteristics of the patients are described in table 1. The median time from transplant to home spirometry monitoring onset was 4.4 months (IQR 3-7.1) and the monitoring was continued for a median time of 16.2 months (IQR 7.2-21.2). According to the criteria published by KugLen et al. [12], good adherence ( $>80 \%$ scheduled home spirometry effectively performed) was observed in $35.5 \%$ patients, moderate adherence $(50-80 \%$ home spirometry performed) in $31.8 \%$ and poor adherence $(<50 \%$ home spirometry performed) in $32.7 \%$. During the follow-up time, 49 drops $\geqslant 20 \%$ in FEV 1 in home spirometry were reported in 34 patients, and $34 / 49(69 \%)$ in 26 patients were confirmed by standard spirometry. 17 drops were related to BOS diagnosis $(n=13)$ or worsening $(n=4)$, but home spirometry anomalies also

@ERSpublications

Long-term outcome of patients with BOS after HSCT with home spirometry is encouraging despite suboptimal adherence http://ow.ly/mKfM30jDknz

Cite this article as: Loiseau $\mathrm{C}$, Lemonnier $\mathrm{F}$, Randrianarivelo $\mathrm{O}$, et al. Home spirometry in bronchiolitis obliterans after allogeneic haematopoietic cell transplant. Eur Respir J 2018; 52: 1702328 [https://doi.org/ 10.1183/13993003.02328-2017]. 
TABLE 1 Characteristics of the population




allowed the detection of other pulmonary complications: infections $(n=6)$, pulmonary embolism $(n=1)$, cardiac failure $(n=3)$ or others $(n=7)$.

26 BOS were diagnosed at a median time of 14 months (IQR 10.5-21.1) post-HSCT, leading to a 5-year cumulative incidence of $27.7 \%$ (95\% CI 19.9-38.8\%). In 13 of the cases, BOS detection was made by home spirometry. Reasons for lack of BOS diagnosis by home spirometry were non-adherence $(n=6)$, BOS occurring before $(n=2)$ or after $(n=4)$ monitoring or non-specified $(n=1)$. Finally, home spirometry detected 13 (65\%) out of 20 BOS occurring during the effective period of equipment: nine (82\%) out of 11 in good/moderate adherers versus four (44\%) out of nine in poor adherers. Thus, home spirometry was a useful tool for BOS detection in good adherers.

At BOS diagnosis, three patients were asymptomatic. The median initial drop in FEV1, compared with pre-transplant data, was 39.9\% (IQR 27.3-45.8). All patients with BOS except one presented extra pulmonary manifestations of chronic GVHD. The 5-year cumulative incidence of BOS was 36.9\% (95\% CI $25.2-48.5 \%)$ in patients with chronic GVHD, compared to $2.4 \%$ (95\% CI 0-7\%) in those who did not ( $p=0.006$ Fine and Gray test). This confirmed that patients with extrapulmonary chronic GVHD have the highest risk of developing BOS, and could represent a group of patients that could benefit from home spirometry.

BOS features were followed for a median of 9.4 years (IQR 7.4-10.7). The median maximal drop in FEV1, compared with pre-transplant data, was $45.8 \%$ (IQR 36.4-58.5). Treatment of BOS consisted in systemic steroids less than $1 \mathrm{mg}$ per $\mathrm{kg}$ per day in $65.4 \%$ patients, associated with other immunosuppressive therapies depending on those previously administrated and on GVHD extrapulmonary organ involvement and specific pulmonary treatment (inhaled corticosteroids and bronchodilatators, and azithromycin) as well as infectious prophylaxis. At last evaluation, worsening of FEV1 (decrease $>15 \%$ compared with diagnosis) was observed in seven $(26.9 \%)$ patients whereas improvement (increase $>15 \%$ ) occurred in eight $(30.8 \%)$ and stabilisation (variation $\leqslant 15 \%$ ) in $11(42.3 \%)$. From the 26 patients with BOS, two died of evolution of BOS associated with respiratory infection leading to an 8 -year mortality due to BOS of $8.1 \%$ (95\% CI $0-19.1 \%)$.

Aiming to indirectly evaluate the home spirometry efficacy, we compared BOS outcome in good/moderate or poor adherers and failed to detect any difference between these patients (table 1). Although this could argue against the home spirometry utility after HSCT, no definitive conclusions can be drawn and only a randomised trial would definitely conclude about the home spirometry usefulness.

We report here with a long follow-up time the features of BOS in a population of HSCT recipients monitored by home spirometry, remarkable by the absence of pejorative effect of BOS occurrence. Indeed, only two of the 26 BOS patients died from respiratory failure, while $73 \%$ patients showed a favourable evolution (FEV1 stable or improved) which has been correlated to a better outcome in post-HSCT BOS [13]. It is noteworthy that BOS incidence, $27.7 \%$, was similar to other studies [14] and FEV1 drop at diagnosis was close to that reported in the NIH series [15] making unlikely the good outcome of BOS patients being related to a lower gravity of BOS.

We showed in this large cohort that home spirometry was feasible in HSCT patients. However, adherence rate was $35.5 \%$, which is lower than the $68 \%$ observed after pulmonary transplantation [12]. This low adherence rate could be a limiting factor for home spirometry use in HSCT patients. In further studies, specific interventions are warranted to improve adherence of these patients less aware of the severity of pulmonary complications than lung transplant patients. These include 1) a better education of patients with dedicated consultations, requiring a close collaboration between haematologists, pulmonologists and patients, 2) development of systems with internet based transmission of home spirometry data and 3) providing reminders to patients through an automated reminding system.

Our study shows that home spirometry allows BOS detection in most adherer patients and raises the issue of a potential favourable impact of home spirometry on post-HSCT BOS outcome. A randomised trial, comparing home spirometry versus absence of home spirometry monitoring in HSCT patients, with specific interventions to improve adherence, would definitively address the interest of this non-invasive procedure on BOS outcome.

Clémence Loiseau $\oplus^{1,10}$, François Lemonnier ${ }^{2,10}$, Odile Randrianarivelo ${ }^{3}$, Raphael Itzykson ${ }^{4}$, Stéphanie Nguyen ${ }^{5}$, Marie Hélène Becquemin ${ }^{3}$, Colas Tcherakian ${ }^{1,6,7}$, Madalina Uzunov ${ }^{5}$, Emilie Catherinot ${ }^{1}$, Elisabeth Rivaud ${ }^{1}$, Hélène Salvator ${ }^{1,6,7}$, Philippe Devillier ${ }^{1,6,7}$, Laurent Sutton ${ }^{8}$, Jean-Paul Vernant ${ }^{5}$, Louis Jean Couderc ${ }^{1,6,7,11}$ and Nathalie Dhédin ${ }^{5,9,11}$

${ }^{1}$ Respiratory Diseases Dept, Foch Hospital, Suresnes, France. ${ }^{2}$ Haematology Dept, Henri Mondor Hospital, Créteil, France. ${ }^{3}$ Functional Pulmonary Test Unit, Pitié Salpêtrière Hospital, Paris, France. ${ }^{4}$ Haematology Dept, Saint-Louis Hospital, Paris, France. ${ }^{5}$ Haematology Dept, Pitié Salpêtrière Hospital, Paris, France. ${ }^{6}$ Faculté des Sciences de la Vie Simone Veil, Université de Versailles St Quentin, Saint Quentin en Yvelines, France. ${ }^{7}$ UPRES EA 220, Suresnes, 
on behalf of the Immunodeficiency Lung Study Group of Foch Hospital, Suresnes, France. ${ }^{8}$ Haematology Dept, Argenteuil Hospital, Argenteuil, France. ${ }^{9}$ Haematology Dept, Adolescents and Young Adults Unit, Saint-Louis Hospital, Paris, France. ${ }^{10}$ Equal contribution as first authors. ${ }^{11}$ Equal contribution as last authors.

Correspondence: Nathalie Dhédin, Hôpital Saint Louis, Service d'Hématologie, Unité Adolescents Jeunes Adultes (AJA), 1 avenue Claude Vellefaux, 75010 Paris, France, nathalie.dhedin@aphp.fr

Received: April 272017 | Accepted after revision: April 152018

Acknowledgement: We thank LVL Medical Company for technical assistance with home spirometry monitoring devices and Polly Gobin for English language revision.

Conflict of interest: E. Catherinot has received funding for meeting attendance from LVL Medical, CSL Behring and LFB, outside the submitted work. H. Salvator has received funding for meeting attendance from GSK and Oxyvie, outside the submitted work.

\section{References}

1 Chien JW, Martin PJ, Gooley TA, et al. Airflow obstruction after myeloablative allogeneic hematopoietic stem cell transplantation. Am J Respir Crit Care Med 2003; 168: 208-214.

2 Bergeron A, Godet C, Chevret S, et al. Bronchiolitis obliterans syndrome after allogeneic hematopoietic SCT: phenotypes and prognosis. Bone Marrow Transplant 2013; 48: 819-824.

3 Soubani AO, Uberti JP. Bronchiolitis obliterans following haematopoietic stem cell transplantation. Eur Respir J 2007; 29: 1007-1019.

4 Rhee $\mathrm{CK}, \mathrm{Ha} \mathrm{JH}$, Yoon $\mathrm{JH}$, et al. Risk factor and clinical outcome of bronchiolitis obliterans syndrome after allogeneic hematopoietic stem cell transplantation. Yonsei Med J 2016; 57: 365-372.

5 Williams KM, Chien JW, Gladwin MT, et al. Bronchiolitis obliterans after allogeneic hematopoietic stem cell transplantation. JAMA 2009; 302: 306-314.

6 Verleden SE, Sacreas A, Vos R, et al. Advances in understanding bronchiolitis obliterans after lung transplantation. Chest 2016; 150: 219-225.

7 Finkelstein SM, Snyder M, Stibbe CE, et al. Staging of bronchiolitis obliterans syndrome using home spirometry. Chest 1999; 116: 120-126.

8 Robson KS, West AJ. Improving survival outcomes in lung transplant recipients through early detection of bronchiolitis obliterans: daily home spirometry versus standard pulmonary function testing. Can J Respir Ther 2014; 50: 17-22.

9 Guihot A, Becquemin M-H, Couderc L-J, et al. Telemetric monitoring of pulmonary function after allogeneic hematopoietic stem cell transplantation. Transplantation 2007; 83: 554-560.

10 Cheng G-S, Campbell AP, Xie H, et al. Correlation and agreement of handheld spirometry with laboratory spirometry in allogeneic hematopoietic cell transplant recipients. Biol Blood Marrow Transplant 2016; 22: 925-931.

11 Meyer KC, Raghu G, Verleden GM, et al. An international ISHLT/ATS/ERS clinical practice guideline: diagnosis and management of bronchiolitis obliterans syndrome. Eur Respir J 2014; 44: 1479-1503.

12 Kugler C, Fuehner T, Dierich M, et al. Effect of adherence to home spirometry on bronchiolitis obliterans and graft survival after lung transplantation. Transplantation 2009; 88: 129-134.

13 Dudek AZ, Mahaseth H, DeFor TE, et al. Bronchiolitis obliterans in chronic graft-versus-host disease: analysis of risk factors and treatment outcomes. Biol Blood Marrow Transplant 2003; 9: 657-666.

14 Chien JW, Duncan S, Williams KM, et al. Bronchiolitis obliterans syndrome after allogeneic hematopoietic stem cell transplantation-an increasingly recognized manifestation of chronic graft-versus-host disease. Biol Blood Marrow Transplant 2010; 16: S106-S114.

15 Abedin S, Yanik GA, Braun T, et al. Predictive value of bronchiolitis obliterans syndrome stage 0p in chronic graft-versus-host disease of the lung. Biol Blood Marrow Transplant 2015; 21: 1127-1131. 